\title{
Haptic interaction becomes reality
}

\author{
Roope Raisamo*, Veikko Surakka, Jukka Raisamo, Jussi Rantala, Jani Lylykangas and Katri Salminen \\ Tampere Unit for Computer-Human Interaction, Department of Computer Sciences, FIN-33014 \\ University of Tampere, Finland
}

\begin{abstract}
Haptic interaction has for a long time been a promise that has not fully been realized in everyday technology due to several reasons. Already for more than 20 years the research community in the field of human-technology interaction has identified multimodal interaction as a potential next mainstream interaction paradigm to replace graphical user interfaces. At the same time, both personal computers and mobile devices have developed rapidly allowing more computing power, more sophisticated feedback through different channels such as display and audio, and more ways of interaction to be used in everyday computing tasks. Within the past few years, haptic interaction has been under rapid research and development. In this article, we will give an introduction to the present state of the art in haptic interaction technology and its promises in mainstream information and communication technology.
\end{abstract}

Keywords: Haptic interaction, cognitive and emotional information, multimodal interaction

\section{Introduction}

The term haptics refers to the field of research exploring human perception and interactions mediated via the sense of touch including hardware and software systems able to provide touch feedback. Haptic communication refers to the use of artificially produced haptic stimulations as a medium for communication between two or more persons. There is a rich potential on how these stimulations can be created. For example, the systems can be made to produce single taps on the skin to grab attention, vibration forces guiding the user while navigating, and even thermal variations to inform of the presence of friends.

Tactile sensations can be produced by several different techniques using, for example, vibrotactile transducers, solenoid-driven pins, piezoelectric actuators or electrotactile stimulators. The stimulus patterns can be modified to form a systematic coding language of some sort. There are only few haptic notation systems developed, such as the widely used Braille system (i.e. language for the visually impaired), but also less acknowledged ones such as Vibratese language [13] designed to transmit single letters and digits as well as the most common English words. These kinds of complex systems, however, require an extensive amount of time and effort to learn, and they are used more as a substitution for than together with the other feedback modalities.

One factor preventing a wider use of haptics in ubiquitous contexts has been the lack of suitable mobile technology for producing haptic feedback. Earlier, virtually only vibration motors with eccentric rotating weights were used in devices, such as mobile phones, to provide coarse haptic feedback to the user. However, the scale of different haptic effects produced with these devices is limited. In addition, the haptic feedback created by vibrating motors is felt across the entire device and thus cannot be presented to a specific location only.

Better actuators capable of producing more finegrained haptic feedback have been developed and used lately. Luk et al. [15] introduced a handheld device with a small tactile display based on piezoelectric actuators. These actuators were used for applying lateral skin stretch to the user's thumb. In another study Poupyrev and Maruyama [18] embedded a piezoelectric actuator inside the touch screen of a handheld device. The haptic feedback was felt right under the current contact point on the display. These techniques are examples of ways to create more ver-

Corresponding author. E-mail: rr@cs.uta.fi. 
satile, fine-grained and localized haptic feedback. This enables more expressive communication as the stimulus patterns can be made more distinguishable.

\section{Research on haptic technology}

Although being a very promising field of research, scientific systematization of haptics is still in its initial stages. The research can roughly be divided in two fields of interest: cognitive (e.g., the type and form of message arriving in the mobile phone) or emotional (e.g., a comforting "hug").

In previous studies mostly cognitive aspects of haptic information have been studied (e.g., Brewster et al. [7]; Rovers \& van Essen [19]). In these studies (Brown et al. [8]; Brewster \& Brown [6]; Brown \& Kaaresoja [9]) first various device prototypes were built and then a set of haptic icons were designed by the researchers themselves (i.e. on the basis of a "hunch" or intuition). These icons with certain meanings have been tested by studying how easily people have been able to learn and identify these haptic messages and icons. The results have been promising. However, the haptic stimuli produced with these devices can be difficult or impossible to reproduce with a different set of hardware. Thus, there is a clear lack of scientific basis for how to systematically support this kind of informative communication. The same holds for the research on the relationship between haptics and emotions.

Although one significant function of touch in human-human interaction is related to human emotion systems (e.g., [14], p. 77-79), most of the current research in human-technology interaction has so far concentrated on the intuitive way of doing things. Admittedly a number of prototypes have been developed for haptic emotional interaction. These devices include, for example, LumiTouch which is a photo frame that turns touch input into flashing lights [10], Hapticat which mimics reactions and purring of a cat [23], TapTap which is a blanket that provides comforting tap of vibration to the shoulder of a user [4], and The Hug, a special pillow which provides a vibrating "hug" to a loved one far apart [11]. These devices are motivated by the idea that touch is an important channel for communication and it can, among other important functions, ease the feeling of social isolation. Users like the elderly and lovers geographically apart from their beloved ones could use these devices for intimate communication.

So far the studies done with these devices have been relatively informal not fulfilling the demands of rigorous scientific investigation. The haptic stimuli they are able to produce are only assumed to be emotional. Also, these stimuli are not device independent. Virtually all experimental evidence for supporting the idea of how to apply emotional and social haptic messages into device independent mobile technology is missing, even though the success at the mobile industry can only rely on systematic scientific evidence. Therefore, carefully planned experimental research on haptics is needed for building grounds for future guidelines on how actually to program the qualities of haptics to technology.

One logical way to proceed is to start to measure emotion related responses to haptic stimulation as such. This could offer one way out in setting aside device specific questions which is very much needed also according to Smith and MacLean [21]. Emotion related reactions can be measured in multiple ways. One can measure low level reactions like pupil size variation, facial electromyography (EMG), ballistocardiography, and more high level responses like ratings of personal emotional experiences with various scales (e.g., Anttonen \& Surakka [1]; Aula \& Surakka [2]; Partala \& Surakka [16]; Partala et al. [17]).

In studying the possible emotional reactions haptics can evoke, it is reasonable first to start with higher level measures. There are basically two approaches in emotion research. One can work with the differential emotions theory in which emotions are seen as distinct categories (e.g. happiness, sadness, anger, surprise) each having their specific motivational properties (e.g., Izard [14]; Ekman [12]; Surakka \& Hietanen [22]). Alternatively one can work with the dimensional view of emotions that maps emotions as combinations of two or more dimensions (e.g., Bradley and Lang [5]; Salminen et al. [20]). These two different approaches can rather be seen as more complementary than contradictory to each other. As argued above, at the moment we are somewhat shorthanded in preplanning discrete emotional messages for information technology. So this is a good topic to start further research in this area.

\section{Haptics in mobile and multimodal systems}

Multimodal interaction refers to utilizing several input and/or output modalities in human-technology interaction. The early studies of Richard Bolt [3] already showed promise of multimodal interaction in making the use of technology more natural, expressive, and efficient. However, even if many research- 
ers have proven benefits of multimodal interaction, it has not become a reality for ordinary users even in 30 years. At the same time, both desktop computers and mobile devices have been developed rapidly, and offer many parallel input and output capabilities. The problem in using multiple modalities has been in that research is still needed to find out how to design multimodal interfaces in such a way that the potential benefits will be available also in practice. When more expressive haptic technology is now becoming available in mobile devices, this makes it possible to have multimodal interfaces become reality. Haptic feedback can be used as a context-dependent alternative or complementary output modality. Contextdependency and modality scheduling assume that the system manages multimodal interaction.

In the present interfaces, haptic and auditory modalities have almost exclusively been used to alarm the user of an incoming call or a scheduled meeting. This kind of use of modalities is very restricted. There are many realistic scenarios in which the sense of touch could be utilized in much more diverse ways.

Haptics could provide a private feedback channel for hand held devices. Imagine that you are on a holiday in a foreign city. You have programmed a sight-seeing route into the GPS navigator in your mobile phone but surely you would rather focus on the glories of the city than flicking constantly the map in the display when walking. Moreover, you would not feel comfortable keeping your expensive phone visible to others, especially when you have been forewarned of bag snatchers who are prowling unsuspecting tourists in the area. Although being a tourist you do not want to look like one. If the navigation system in your phone was equipped with novel haptic feedback features, the device could be held firmly in hand, and your hand put in a pocket. You could feel intuitive navigation instructions in your palm privately and inconspicuously while walking like you were born and bread in the city.

In some situations, haptic feedback might also be the only effective way to signal important information for the user. Think, for example, an aged person who has low vision and low hearing ability, and who is recovering from a heart surgery at home needing a continuous heart monitoring to avoid complications. In this case, an efficient instrument designed to prevent the patient to strain the heart too much, would be a wearable or mobile heart rate monitoring device which could warn the patient by haptic sensation when the pulse or blood pressure is in danger to rise too high.
Another example of using haptics is a situation where you are trekking in the woods on a sunny and cold winter day with your heart rate monitor in your wrist. You want to keep your heart rate in a previously set target zones to carry out your exercise effectively and safely. However, the temperature is $-20^{\circ} \mathrm{C}$ and you would not feel happy to drag out your wrist under many sleeve layers and mitten every now and then to see how is your pulse, especially because the screen is difficult to read in the bright sunshine. You would not want to break the restful silence of wild nature by disturbing audible alerts either. Or what if you are jogging and a heavy traffic lap falls in your path. You know that it is not safe to watch the screen in your wrist in order to avoid stumbling or banging into others in the street. Due to heavy traffic it would be difficult to hear the alarms too. In these kinds of situations it would be nice to get the heart rate information, when necessary, in a gentle and sophisticated way via haptic sensations felt in the skin under the heart rate monitor.

There are many other possibilities to use the sense of touch in human-technology interaction (HTI), but more basic and applied research is needed to find out how to use it. In our view the future user interfaces will be multimodal. Multimodality allows the users to use the ways of interaction that best fit in their abilities and the present context of use. Haptics makes it possible for all users, including people with special needs, to better interact with the technology. Moreover, any user can have a need to operate a mobile device without using the vision. This may be due to the fact that the context of use requires one to draw the visual attention to other cues, or the user may simply choose to do so because it is fun.

\section{Haptic interaction becoming mainstream}

Haptic feedback devices have been known to be clumsy, heavy, and expensive in desktop computers. Some cheap devices have been available for gaming, but they have not been widely used in other applications. It looks like the new devices now appearing in the consumer price category might become common and bought at least by gamers, but this is not sure as the price of these devices is still a few hundred dollars. When the devices are available in large enough quantities, and bought by the consumers, then the consumer technology is ready to support 3D haptic interaction in different kinds of software. However, it may well be that even if haptic feedback has been 
available in desktop systems for much longer than in mobile systems, desktop systems are not the ones that make haptic interaction widely available for the general public.

Haptic input has been used in mobile computing for a long time in the form of simple vibration alarms. However, the introduction of touchscreens really increased the interest in haptic feedback. An example of the need for haptic feedback is the popular Apple iPhone that does not presently have haptic feedback produced on the screen when it is touched. The lack of haptic feedback makes it necessary to look at the device when interacting with it, which may not be the best choice in all situations when mobile. Apple is not alone with this problem. Several mobile phone manufacturers such as Nokia, Sony-Ericsson, Samsung, and Motorola, have lately shown increasing interest in creating touch-based user interaction that also makes use of more advanced haptic feedback technology, such as accurately controlled vibrotactile actuators or piezoelectric actuators.

Making more expressive haptic interaction available for all users is already a big step in providing the user with more interaction possibilities depending on context, and the developer with more interaction design possibilities when different modalities are combined. In our view, adding haptic technology in mobile devices may act as a catalyst in making truly multimodal interaction available in mobile devices. This view is based on the fact that haptics is best used either to support the other modalities in the same task or to provide a context-aware interaction modality when, for example, sight or hearing cannot be used due to environmental conditions or needs to focus on something else. This is why adding haptic interaction requires the device and the software to be able to manage multimodal interaction, and thus other modalities can be supported at the same time. If this happens, as we expect, we can soon have adaptive, context-aware, and even intelligent mobile applications in different mobile devices used by all.

\section{Summary}

In this article, we have discussed the present state of the art in haptic interaction technology. We addressed both cognitive and emotional aspects of haptic technology. Several potential examples of the use of haptics were given, and in most cases the use of haptics was a part of context-aware multimodal system. The scientific work and commercial development in this area is still just in the beginning, but it is already visible that expressive haptic interaction will become a reality within a few years. When haptic technology will be widely available, it has potential for increasing the efficiency, adaptivity and expressiveness of human-technology interaction. Then, the benefits found in the research on multimodal interaction will finally be realized in every-day computing.

\section{Acknowledgments}

We thank the research and development community that has made it possible to actually produce haptic feedback in so many new ways and thus has enabled studying and using of haptic interaction. This work was partially funded by the Finnish Funding Agency for Technology and Innovation (Tekes), decision 40120/08. More information is available through the web at http://mobilehaptics.cs.uta.fi/en/.

\section{References}

[1] J. Anttonen and V. Surakka, Emotions and heart rate while sitting on a chair. In Proc. ACM SIGCHI Conference on Human Factors in Computing Systems (CHI 2005), ACM Press, NY, 2005, pp. 491-499.

[2] A. Aula and V. Surakka, Auditory emotional feedback facilitates human-computer interaction. In People and Computers XVI: Memorable yet Invisible, Proceedings of HCI 2002. Springer-Verlag, London, 2002, pp. 337-349.

[3] R.A. Bolt, Put-that-there. In Proceedings of SIGGRAPH '80, ACM Press, 1980, pp. 262-270.

[4] L. Bonnani, C. Lieberman, J. Vaucelle, and O. Zuckerman, TapTap: A haptic wearable for asynchronous distributed touch therapy. In Proc. ACM SIGCHI Conference on Human Factors in Computing Systems (CHI 2006), ACM Press, NY, 2006, pp. 580-585.

[5] M. Bradley and P.J. Lang, Measuring emotion: The selfassessment manikin and the semantic differential. Journal of Behavioral Therapy \& Experimental Psychiatry 25 (1), 1994, 49-59.

[6] S.A. Brewster and L.M. Brown, Tactons: Structured tactile messages for non-visual information display. In Proc. The Australasian User Interface Conference (AUIC'04), Australian Computer Society, 2004, pp. 15-23.

[7] S.A. Brewster, F. Chohan, and L.M. Brown, Tactile Feedback for Mobile Interactions. In Proc. ACM SIGCHI Conference on Human factors in Computing Systems (CHI 2007), ACM Press, NY, 2007, pp. 159-162.

[8] L.M. Brown, S.A. Brewster, and H. Purchase, H., A first investigation into the effectiveness of Tactons. In Proc. First Joint Eurohaptics Conference and Symposium on Haptic Interfaces for Virtual Environment and Teleoperator Systems (WorldHaptics 2005), IEEE Press, 2005, pp. 167-176.

[9] L.M. Brown, and T. Kaaresoja, Feel who's talking: Using tactons for mobile phone alerts. In Proc. Extended Abstracts on Human Factors in Computing Systems (CHI 2006), ACM Press, NY, 2006, pp. 604-609.

[10] A. Chang, B. Resner, B. Koerner, X.C. Wang, and H. Ishii, LumiTouch: An emotional communication device. In Proc. 
ACM SIGCHI Conference on Human factors in Computing Systems (CHI 2001), ACM Press, NY, 2001, pp. 313-314.

[11] C. DiSalvo, F. Gemperle, J. Forlizzi, and E. Montgomery, The hug: An exploration of robotic form for intimate communication. In CD-ROM Proc. IEEE International Workshop on Robot and Human Interactive Communication (ROMAN 2003), IEEE Press, 2003, pp. 403-408.

[12] P. Ekman, Strong evidence for universals in facial expressions: A reply to Russell's mistaken critique. Psychological Bulletin 115, 1994, pp. 268-287.

[13] F.A. Geldard, Adventures in tactile literacy. The American Psychologist 12, 1957, pp. 115-124.

[14] C.E. Izard, Human Emotions. Plenum Press, New York, NY, 1977.

[15] J. Luk, J. Pasquero, S. Little, K.E. MacLean, V. Levesque, and V. Hayward, A role for haptics in mobile interaction: initial design using a handheld tactile display prototype. In Proc. ACM SIGCHI Conference on Human Factors in computing systems (CHI 2006), ACM Press, NY, 2006, pp. 171-180.

[16] T. Partala and V. Surakka, The effects of affective interventions in human-computer interaction. Interacting with Computers 16 (2), 2004, pp. 295-309.

[17] T. Partala, V. Surakka, and T. Vanhala, Real-time estimation of emotional experiences from facial expressions. Interacting with Computers 18 (2), 2006, pp. 208-226.
[18] I. Poupyrev and S. Maruyama, Tactile interfaces for small touch screens. In Proc. Symposium on User Interface Software and Technology (UIST 2002), ACM Press, NY, 2002, pp. $217-220$.

[19] A.F. Rovers, and H.A. van Essen, Using active haptic feedback in everyday products. In Proc. EuroHaptics 2006, Springer-Verlag, 2006, pp. 447-453.

[20] K. Salminen, V. Surakka, J. Lylykangas, J. Raisamo, R. Saarinen, J. Rantala, R. Raisamo, and G. Evreinov, Emotional and Behavioral Responses to Haptic Stimulation. In Proc. ACM SIGCHI Conference on Human Factors in Computing Systems (CHI 2008), ACM Press, NY, 2008, pp. $1555-1562$.

[21] J. Smith and K.E. MacLean, K. E., Communicating emotion through a haptic link: Design space and methodology. International Journal of Human-Computer Studies 65 (4), 2007. pp. 376-387.

[22] V. Surakka and J.K. Hietanen, Facial and emotional reactions to Duchenne and non-Duchenne smiles. International Journal of Psychophysiology 29, 1998, pp. 23-33.

[23] S. Yohanan, M. Chan, J. Hopkins, H. Sun, and K.E. MacLean, Hapticat: exploration of affective touch. In Proc. International Conference on Multimodal Interfaces (ICMI 2005), ACM Press, NY, 2005, pp. 222-229. 